02,05

\title{
Вихревая структура ВТСП в неоднородном магнитном поле
}

\author{
(C) А.Н. Максимова, В.А. Кашурников, А.Н. Мороз, И.А. Руднев \\ Национальный исследовательский ядерный университет „МИФИ“, \\ Москва, Россия \\ E-mail: anmaksimova@mephi.ru
}

Поступила в Редакцию 22 октября 2020 г.

В окончательной редакции 22 декабря 2020 г.

Принята к публикации 3 января 2020 г.

\begin{abstract}
Численно исследовано проникновение магнитного потока в слоистый анизотропный сверхпроводник, помещенный в градиентное магнитное поле, эквивалентное полю постоянного магнита. Рассчитаны вихревые конфигурации, возникающие как в бездефектном сверхпроводнике, так сверхпроводнике, содержащем дефекты. Показано, что проникновение неоднородного магнитного поля в слоистый сверхпроводник происходит через формирование неполных вихревых нитей на границе, что означает появление параллельной слоям компоненты магнитного поля. Рассчитаны зависимости намагниченности и энергии в магнитном поле для трехмерного образца ВТСП, показано существование минимумов на графике энергии, соответствующих устойчивым положениям магнита относительно сверхпроводника.
\end{abstract}

Ключевые слова: ВТСП, вихри Абрикосова, метод Монте-Карло, магнитная левитация.

DOI: 10.21883/FTT.2021.05.50806.226

\section{1. Введение}

Несмотря на огромное количество проведенных исследований, изучение влияния магнитного поля на сверхпроводник второго рода и в настоящее время представляет существенный научный и практический интерес [1-2]. Различные эффекты, такие как рост критического тока с увеличением магнитного поля, что проявляется в уширении петли перемагничивания образца [2], практически полностью определяются структурой и динамикой вихревой решетки. Сверхпроводящие образцы, а также кабели и ленты на основе ВТСП используются в сверхпроводящих магнитах [3], ограничителях тока $[4,5]$ и в левитационной технологии [6-8]. В последнем случае используется магнитолевитационная система сверхпроводник - постоянный магнит, в которой сверхпроводник находится в градиентном поле постоянного магнита. Такая ситуация по распределению магнитного поля внутри сверхпроводника, так же как и по намагниченности сверхпроводника принципиально отличается от картины с однородным полем. Однако, практически важные характеристики образца и в этом случае определяются динамикой вихревой решетки. Поэтому важным представляется исследование влияния неоднородного магнитного поля, создаваемого реальным магнитом, на вихревую систему и, как следствие, на намагниченность и транспортные характеристики сверхпроводника. Целью настоящей работы является анализ влияния градиента магнитного поля на вихревую систему ВТСП, выявление особенностей, обусловленных неоднородностью внешнего магнитного поля. Для исследования нами был выбран Ві-2212, отличающийся высокой анизотропией и, как следствие, существенным разнообразием вихревых структур в магнитном поле, что означает ярко выраженный отклик на изменение внешних параметров.

Высокотемпературные сверхпроводники, в большинстве случаев используемые на практике, являются слоистыми анизотропными веществами, вихревая нить в таких сверхпроводниках может быть представлена в виде стопки квазидвумерных слоевых вихрей - панкейков. Модель вихревой решетки в слоистом ВТСП построена в работах [9-11], где получены соотношения для магнитного поля, экранирующих токов и энергии уединенного панкейка в стопке сверхпроводящих плоскостей, прямой и наклонной вихревой нити. В работах [12,13], обзор [14] и ссылки в нем, [15,16] исследован важный случай, когда магнитное поле составляет некоторый угол с осью анизотропии. Наряду с абрикосовскими вихрями в такой системе присутствуют джозефсоновские вихри, рождающиеся под действием компоненты магнитного поля, параллельной слоям, что дополнительно усложняет фазовую диаграмму. Одним из интересных эффектов, продемонстрированных в этих работах, является притяжение между наклонными вихревыми нитями.

Исследования, связанные с градиентным магнитным полем, в настоящее время носят в основном экспериментальный характер. В работе [17] систематически исследовались магнитные свойства стопки ВТСП-лент, получены кривые намагниченности при различной температуре и числе лент $N$ в стопке, измерена сила левитации. Подробнее аналогичные данные получены также для отдельных ВТСП-лент и стопок ВТСП-лент, представляющих собой наборную конструкцию с многочисленными слоями ВТСП [18-21]. Показано, что величина остаточной намагниченности выходит на насыщение, а скорость релаксации остаточной намагниченности падает при увеличении $N$. В серии работ $[22,23]$ 
разработан метод для расчета кривых намагниченности и силы левитации сверхпроводника второго рода, помещенного в однородное и неоднородное магнитное поле. В работе [24] выполнены измерения силы левитации для тонкого сверхпроводящего слоя и экспериментально исследовано проникновение магнитного потока в образец. В работе [25] также аналитически рассчитана сила левитации для сверхпроводящего диска и цилиндрического постоянного магнита. Но стоит отметить, что в перечисленных работах отсутствует детальный анализ магнитного потока в градиентном поле. Также использованные в данных работах методы не дают возможность проанализировать влияние произвольной конфигурации дефектов на силу левитации и проникновение магнитного потока. Поэтому целью настоящей работы является моделирование вихревой решетки слоистого анизотропного ВТСП, находящегося в неоднородном магнитном поле, а также расчет намагниченности и энергии сверхпроводника в поле постоянного магнита.

Статья построена следующим образом. Во Введении кратко описывается проблематика темы статьи, в разделе 2 описывается метод расчета, в разделе 3 представлены результаты расчета для одной сверхпроводящей плоскости и стопки сверхпроводящих плоскостей, основные выводы приводятся в Заключении.

\section{2. Модель и метод расчета}

Расчеты выполнены методом Монте-Карло в рамках трехмерной модели слоистого ВТСП [26,27], основанной на модели Лоренса-Дониака [28]. В рамках данной модели сверхпроводящие слои слабо скоррелированы, и вихревая нить может быть представлена в виде стопки взаимодействующих слоевых вихрей - панкейков, связанных межплоскостным взаимодействием (рис. 1, 2,a). В наших расчетах мы не учитываем параллельную слоям компоненту магнитного поля и рассматриваем слоистый анизотропный сверхпроводник в магнитном поле, параллельном оси анизотропии. Это обусловлено малостью указанной компоненты поля на малых расстояниях от магнита и позволяет избежать излишнего усложнения расчета. Потенциал Гиббса системы квазидвумерных вихрей в плоскости ВТСП-слоя записывается в виде

$$
\begin{gathered}
G=\sum_{z}\left\{N_{z} \varepsilon+\sum_{i<j} U_{\text {in-plane }}\left(r_{i j}\right)+\sum_{i, N_{p}} U_{p}\left(r_{i p}\right)\right. \\
\left.+\sum_{i, j} U_{\text {surf }}\left(\mathbf{r}_{i}, \mathbf{r}_{j}^{(\text {image })}\right)\right\}, \\
U_{\text {in-plane }}\left(r_{i j}\right)=d \frac{\Phi_{0}^{2}}{8 \pi^{2} \lambda^{2}} K_{0}\left(\frac{r_{i j}}{\lambda}\right), \\
U_{\text {surf }}\left(\mathbf{r}_{i}, \mathbf{r}_{j}^{\text {(image })}\right)=-d \varepsilon_{0} K_{0}\left(\frac{\left|r_{i}-r_{j}^{(\text {image })}\right|}{\lambda}\right),
\end{gathered}
$$

$$
U_{m}=d \frac{\Phi_{0}}{4 \pi}\left(H_{0}\left(\frac{\operatorname{ch} \frac{x}{\lambda}}{\operatorname{ch} \frac{L x}{2 \lambda}}-1\right)-H_{I}\left(\frac{\operatorname{sh} \frac{x}{\lambda}}{\operatorname{sh} \frac{L x}{2 \lambda}} \mp 1\right)\right),
$$

где $\varepsilon=d \varepsilon_{0}(\ln [\lambda(T) / \xi(T)]+0.52)-$ собственная энергия вихря, $\xi(0)$ - длина когерентности при $T=0 ; N_{z}-$ число панкейков в плоскости $z$; второй член описывает попарное взаимодействие вихрей внутри плоскости, третий - взаимодействие вихрей с центрами пиннинга, четвертый - с поверхностью и мейсснеровским током; $r_{i j}, r_{i p}$ - модули радиус-векторов, соединяющих два вихря и вихрь с центром пиннинга соответственно, $\varepsilon_{0}=\Phi_{0}^{2} /(4 \pi \lambda)^{2}$, где $\Phi_{0}=\pi \hbar c / e-$ квант магнитного потока, $d$ - толщина сверхпроводящего слоя, $K_{0}\left(r_{i j} / \gamma\right)-$ функция Макдональда.

Взаимодействие вихревой нити с центром пиннинга (дефектом) может быть записано в виде

$$
U_{p n}=-\alpha \frac{1}{1+r_{i p} / \xi} \exp \left(-\frac{r_{i p}}{2 \xi}\right),
$$

где $\alpha-$ эффективная глубина потенциальной ямы дефекта, $N_{p}$ - количество центров пиннинга в образце. В нашей работе предполагается, что дефекты имеют размер порядка радиуса кора вихря $(\xi), \alpha \sim 0.01 \mathrm{eV}$ на один сверхпроводящий слой.

Для описания трехмерной системы вихрей необходимо добавить слагаемое, ответственное за межплоскостное взаимодействие. Межплоскостное взаимодействие панкейков является суммой электромагнитного

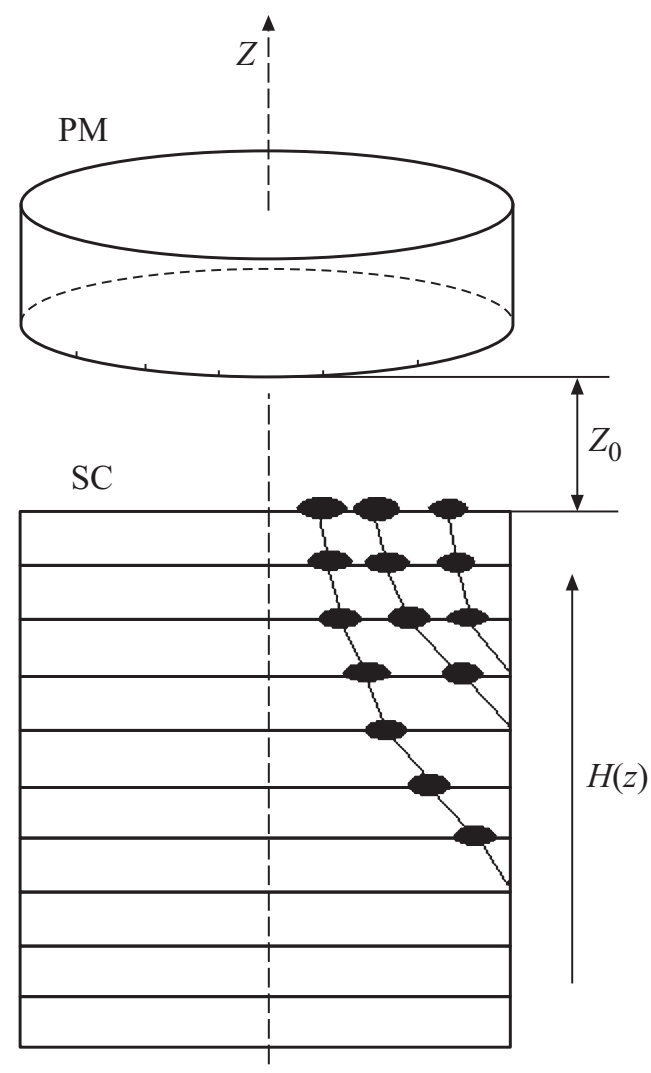

Рис. 1. Геометрия расчета. 
$a$

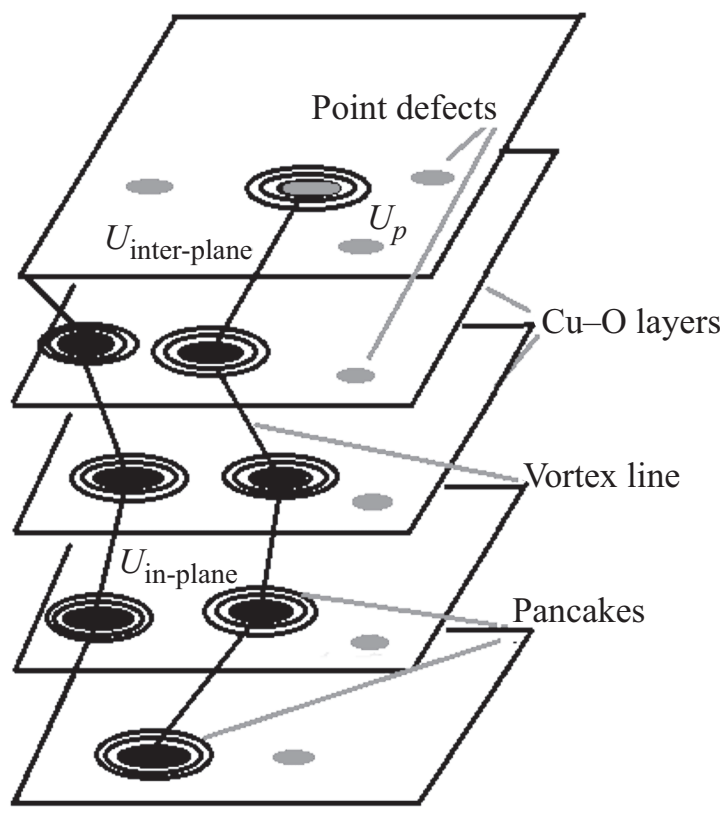

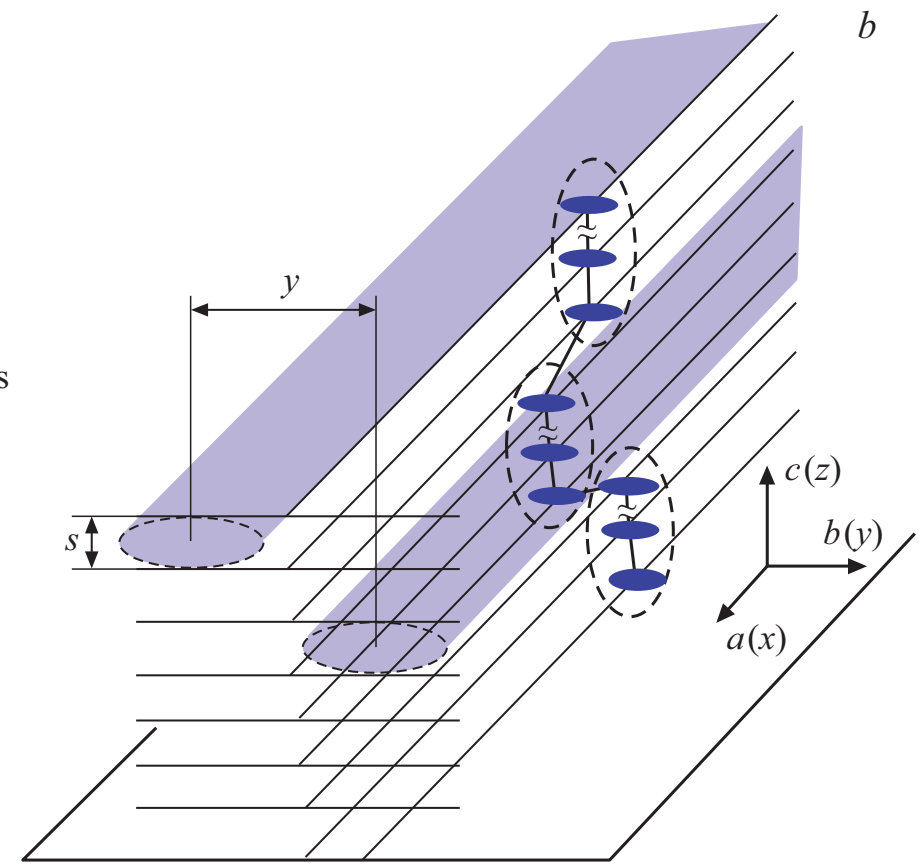

Рис. 2. a) Вихри в слоистом сверхпроводнике. $b$ ) Геометрия расчета для $N_{\mathrm{L}} \sim 1000$ сверхпроводящих плоскостей. Стопки панкейков, принятые за 1 элементарный объект, обведены пунктиром. Серым цветом показаны джозефсоновские вихри.

и джозефсоновского взаимодействий. Электромагнитное взаимодействие $U_{e m}$ связано с взаимодействием панкейка в слое $i$ с токами, наведенными в этом слое панкейками, находящимися в других слоях. Джозефсоновское взаимодействие $U_{j o s}$ обусловлено джозефсоновским током, текущим между сверхпроводящими плоскостями, разделенными изолятором; этот ток пропорционален разности фаз сверхпроводящих волновых функций. Это взаимодействие квадратично при относительных смещениях панкейков, меньших $\gamma s$, и линейно, пропорционально длине джозефсоновской струны, при смещениях, больших $\gamma s$, здесь $s-$ расстояние между сверхпроводящими слоями, $\gamma$ - параметр анизотропии. Выражения для межплоскостного взаимодействия панкейков получены в работах $[29,30]$ и имеют вид

$$
\begin{aligned}
& U_{\text {int } e r-p l a n e}\left(r_{i}^{z, z+1}\right)=U_{e m}\left(r_{i}^{z, z+1}\right)+U_{j o s}\left(r_{i}^{z, z+1}\right), \\
& U_{e m}\left(r_{i}^{z, z+1}\right)=2 s \varepsilon_{0}\left[C+\ln \left(r_{i}^{z, z+1} / 2 \lambda\right)+K_{0}\left(r_{i}^{z, z+1} / \lambda\right)\right], \\
& U_{j o s}^{z, z+1}\left(r_{i}^{z, z+1}\right) \\
& =\left\{\begin{array}{rlrl}
\varepsilon_{0} d[1+\ln (\lambda / s)] 0 & 25\left(r_{i}^{z, z+1} / r_{g}\right)^{2} & \\
\times \ln \left(9 r_{g} / r_{i}^{z, z+1}\right), & & r_{i}^{z, z+1} \leq 2 r_{g}, \\
\varepsilon_{0} d[1+\ln (\lambda / s)]\left[\left(r_{i}^{z, z+1} / r_{g}\right)-0.5\right], & r_{i}^{z, z+1}>2 r_{g},
\end{array}\right.
\end{aligned}
$$

где $C=0.5772$ - постоянная Эйлера, $r_{g}=\gamma s-$ характерное расстояние джозефсоновского взаимодействия. Подробное описание модели и метода расчета см. [26]. В настоящей работе ограничимся случаем магнитного поля, перпендикулярного сверхпроводящим плоскостям. В этом случае в сверхпроводнике реализуется вихревая решетка, состоящая из абрикосовских вихрей. Расчеты выполнены для характерных параметров висмутовых ВТСП $\left(\mathrm{Bi}_{2} \mathrm{Sr}_{2} \mathrm{CaCu}_{2} \mathrm{O}_{8+\delta}\right.$ : толщина сверхпроводящего слоя $d=0.27 \mathrm{~nm}, \lambda(T=0)=180 \mathrm{~nm}$, $\xi(T=0)=2 \mathrm{~nm}, T_{c}=84 \mathrm{~K}, s=2.7 \mathrm{~nm}$. Размер образца в плоскости $a b$ составляет $5 \times 3 \mu \mathrm{m})$ континуальным методом Монте-Карло, с учетом процессов рождения, уничтожения, движения абрикосовских вихрей. Использованный в расчете размер образца много меньше размеров реальных сверхпроводников, используемых на практике. Однако, как показывают оценки и расчеты, в ряде случаев этого размера оказывается достаточно для по крайней мере качественного соответствия эксперименту. Прямой расчет величины критического тока при различной ширине образца показывает, что при слабом пиннинге можно добиться и количественного совпадения с экспериментом.

По этой же причине расчет Монте-Карло для стопки сверхпроводящих плоскостей возможен только при небольшом ( 10) числе плоскостей. С другой стороны, поле постоянного магнита спадает с ростом расстояния $r$ до магнита как $1 / r^{3}$, так что на расстояниях порядка размера стопки из 10 плоскостей неоднородность поля будет крайне мала. Для реализации расчета приближенно можно рассматривать стопку из $\sim 100$ панкейков как единый объект, так что элементарным подпроцессом является перемещение, рождение или уничтожение стопки из 100 панкейков как едино- 

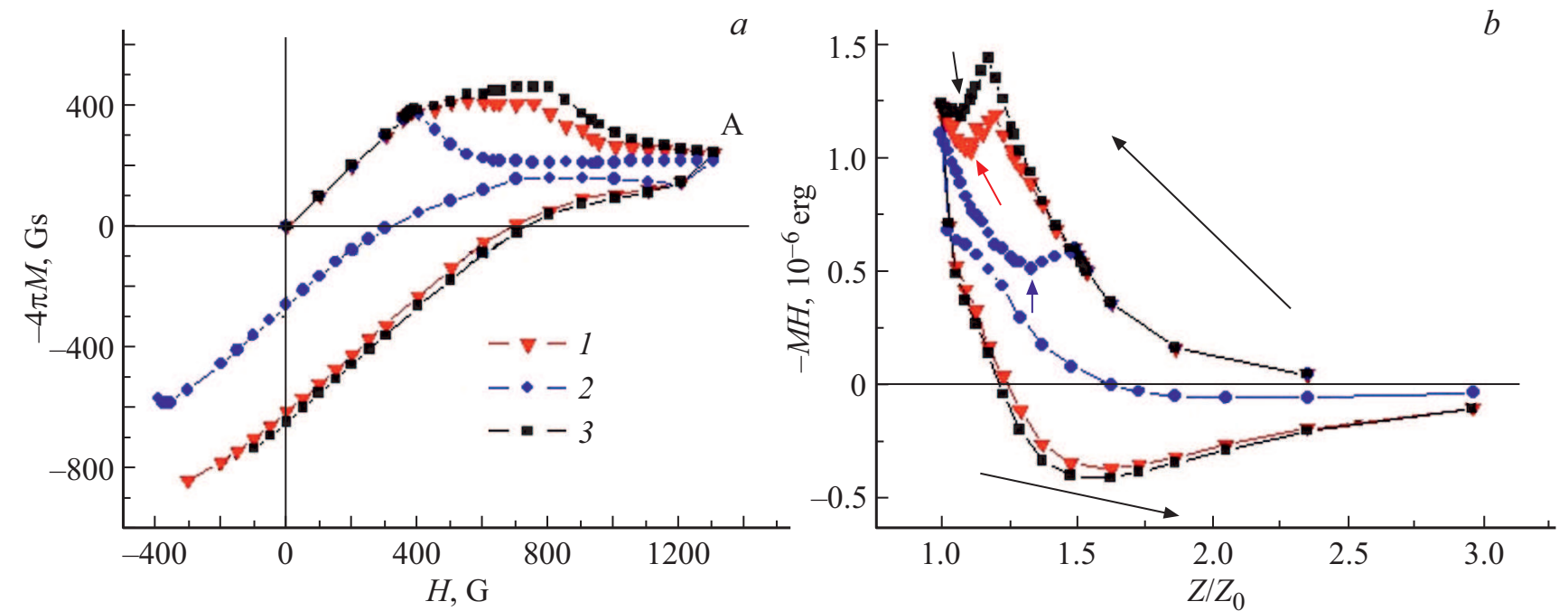

Рис. 3. а) Кривые намагниченности при различных параметрах дефектов. $1-N_{\mathrm{d}}=1000, \alpha=0.01 \mathrm{eV}, 2-N_{\mathrm{d}}=300, \alpha=0.1 \mathrm{eV}$, $3-N_{\mathrm{d}}=1000, \alpha=0.1 \mathrm{eV}$. Расчет сделан для одной сверхпроводящей плоскости, однако увеличение внешнего поля эквивалентно уменьшению расстояния от сверхпроводника до магнита. $b$ ) величина $-M H$, пропорциональная энергии сверхпроводника в магнитном поле. По горизонтальной оси отложено относительное расстояние до магнита. $z_{0}$ - произвольное фиксированное расстояние. Сверхпроводник приближается из бесконечности к магниту на расстояние $z_{0}$, а затем удаляется от него, что показано на рисунке стрелками. Магнитное поле в точке $z_{0}$ совпадает с магнитным полем в точке А на рис. 2,a. Жирными стрелками показаны минимумы энергии, соответствующие равновесным положениям магнита относительно сверхпроводника.

го целого (геометрия расчета показана на рис. $2, b$ ). Таким образом, вихревая нить по-прежнему состоит из $\sim 10$ элементарных объектов. Приближенно такой подход оправдан, поскольку в большинстве расчетов и экспериментов не наблюдается множественных взаимных смещений панкейков. Кроме того, реальные масштабы изгибов абрикосовских вихрей много больше межслоевого расстояния $s$, и определяются параметрами упругости вихревой нити [31]. Для анализа влияния неоднородного магнитного поля на вихревую решетку нами были выполнены расчеты кривых намагниченности при различных параметрах системы. При расчете кривой намагниченности каждая точка петли рассчитывается конечное число шагов Монте-Карло, так, что каждое конечное состояние является начальным для следующего. Такой подход, как показывает большинство наших предыдущих расчетов, позволяет проследить эволюцию системы при изменении внешнего поля.

Для более полного отражения особенностей, связанных с неоднородным магнитным полем, в расчете допускается рождение на границе неполной вихревой нити (рис. 1). В настоящей работе предполагается, что ось симметрии постоянного магнита совпадает с осью анизотропии сверхпроводника и перпендикулярна сверхпроводящим плоскостям (ось $z$ на рис. 1). Неоднородность поля в плоскости, перпендикулярной оси $z$, в расчете не учитывается. Процессы рождения, уничтожения и движения панкейков в плоскостях осуществляются с учетом поля $H(z)$, соответствующего каждой сверхпроводящей плоскости (см. рис. 1).

\section{3. Результаты}

\section{1. Расчет для одной сверхпроводящей плоскости}

Приведем результаты расчета, выполненного в рамках двумерной модели слоистого ВТСП, что соответствует тонкой сверхпроводящей пленке, помещенной в магнитное поле. На рис. 3 показаны кривые намагниченности, рассчитанные в рамках данной модели. Кривые рассчитаны при различных параметрах дефектов и различной неоднородности магнитного поля. Точки на зависимости энергии от расстояния до магнита (рис. $3, b$ ) рассчитаны как произведение магнитного момента на напряженность внешнего поля в соответствующей точке кривой намагниченности (рис. 3,a) Видно, что на всех трех кривых (рис. $3, b$ ) присутствуют минимумы (отвечающие равновесным положениям сверхпроводника) при движении образца к магниту, а на кривых 1 и 3 - минимумы также при движении от магнита.

\section{2. Расчет для стопки сверхпроводящих плоскостей}

Проанализируем теперь поведение вихревой решетки в трехмерном анизотропном сверхпроводнике.

Включение неоднородного магнитного поля неизбежно приводит к особенностям поведения вихревой решетки, не характерным для однородного поля. Проанализируем особенности проникновения магнитного потока с границы сверхпроводника в неоднородном магнитном поле и сравним с проникновением вихрей с границы 


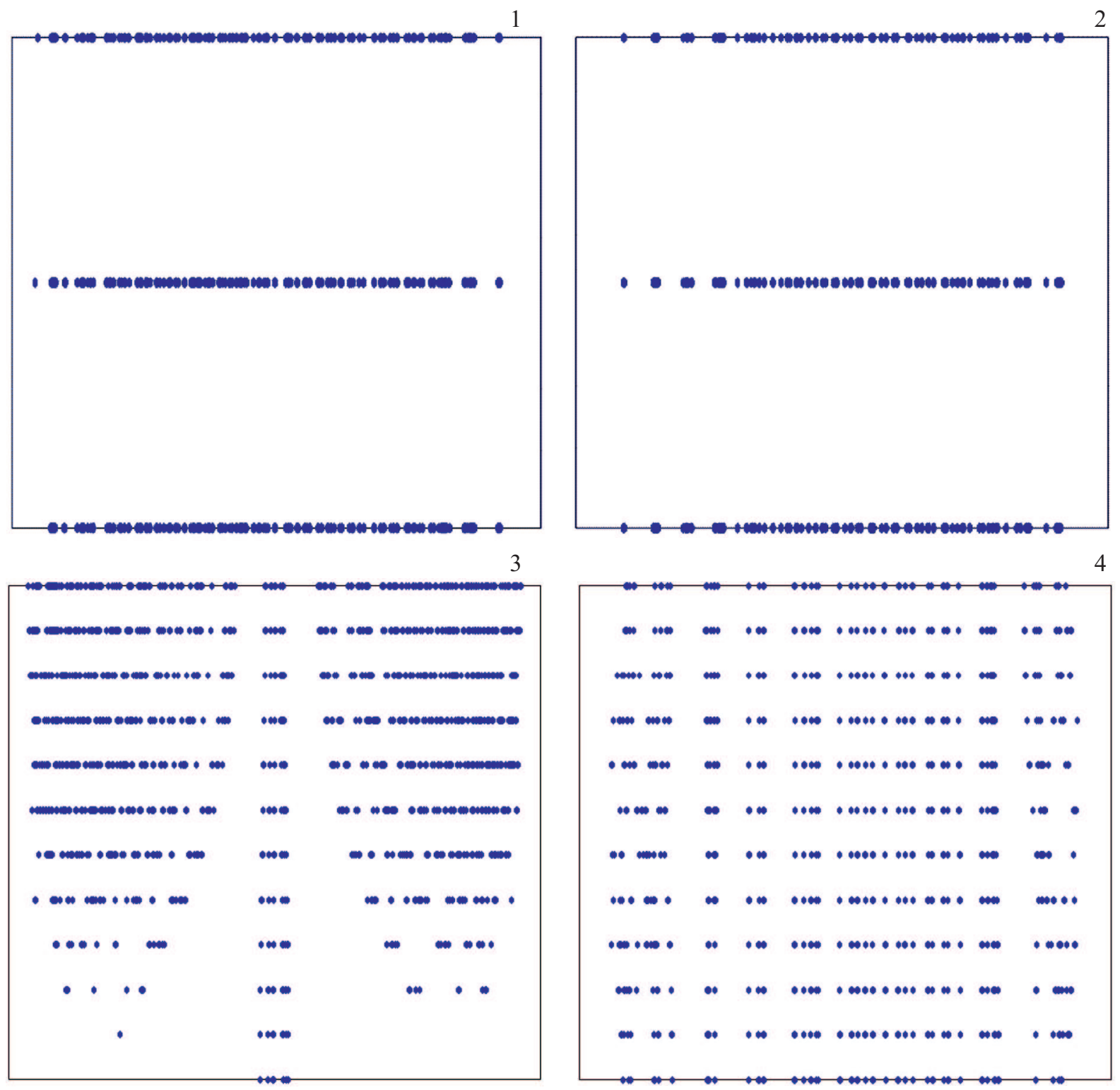

Рис. 4. Проникновение вихрей в бездефектный образец. 1) 3 слоя, неоднородное поле, $H=450 \mathrm{Gs}, 2) 3$ слоя, однородное поле, $H=450 \mathrm{Gs}, 3) 12$ слоев, неоднородное поле, $H=450 \mathrm{Gs}, 4) 12$ слоев, однородное поле, $H=450$ Gs. Кружками обозначены панкейки.

в однородном поле. Введем в модель неоднородность внешнего магнитного поля $H$ в виде зависимости величины $H$ от номера сверхпроводящего слоя (слои нумеруются начиная с нижнего слоя на рис. 1). Предположим, что данная зависимость может быть представлена в виде $H(i)=H(1)(1+i n h \cdot(i-1)), i-$ номер слоя, inh безразмерный параметр, характеризующий неоднородность поля. inh $\sim 0.01$ соответствует неоднородности поля $d H / d z \sim 10^{5} \mathrm{Gs} / \mathrm{cm}$, что достижимо на близких расстояниях от магнита. Заметим, что в данной модели неоднородность зависит от величины поля, соответствующего нижнему сверхпроводящему слою.

Для анализа особенностей проникновения магнитного потока рассмотрим модельную ситуацию, когда $i n h=0.1$. Смоделируем перемагничивание трехмерного образца (см. кривую намагниченности на рис. 3) в неоднородном поле; напряженность поля, отложенная по горизонтальной оси, совпадает с $H(1)$. Рассмотрим случай чистого и дефектного образца. На рис. 4 и 5 показаны схематично вихревые конфигурации, возникающие в бездефектном образце (рис. 4) и в образце с дефектами (рис. 5; концентрация дефектов $c=2 \cdot 10^{9} \mathrm{~cm}^{-2}$ ) при росте $H(1)$. Конфигурации показаны для $H(1)=450 \mathrm{Gs}$ и $N_{\mathrm{L}}=12$ сверхпроводящих слоев. Для сравнения показаны аналогичные конфигурации для однородного поля. Отметим, что в неоднородном поле возможно рождение отрезка вихревой нити, даже если его дальние от магнита панкейки находятся в поле, меньшем первого кри- 


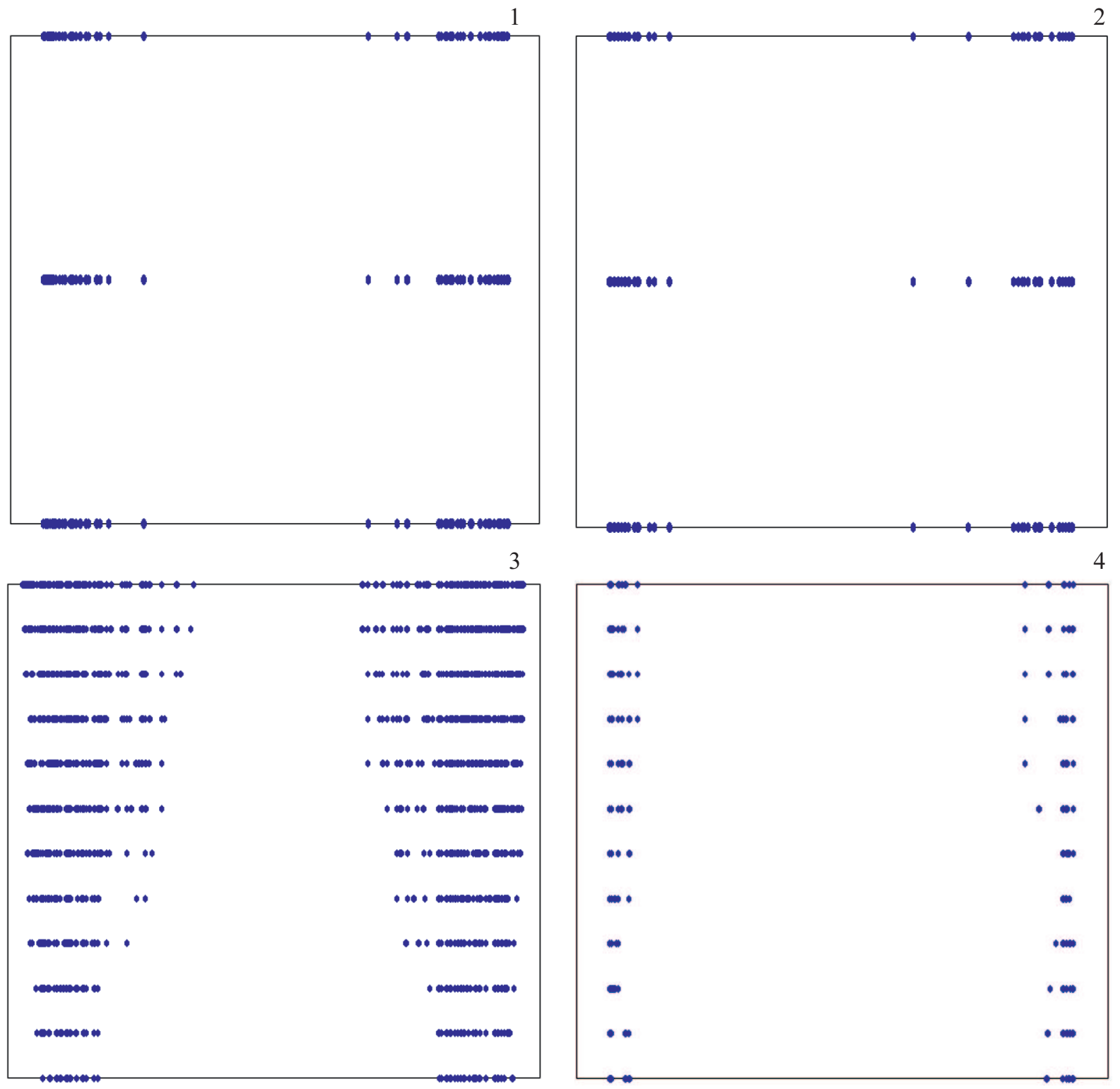

Рис. 5. Проникновение вихрей в дефектный образец. 1) 3 слоя, неоднородное поле, $H=450 \mathrm{Gs}, 2) 3$ слоя, однородное поле, $H=450 \mathrm{Gs}$, 3) 12 слоев, неоднородное поле, $H=450 \mathrm{Gs}$, 4) 12 слоев, однородное поле, $H=450$ Gs. Кружками обозначены панкейки. Повышенная концентрация вихрей на рис. 3 является особенностью, характерной для неоднородного поля, и связана с повышением в неоднородном поле вероятности рождения неполной нити.

тического. Это связано с понижением полной энергии стопки панкейков за счет слагаемого (4). Именно такая ситуация и реализуется на рис. 5 (3). На рис. 6 показаны также вихревые конфигурации в чистом образце, возникающие при движении вдоль кривой намагниченности в сторону увеличения внешнего магнитного поля. В качестве иллюстрации к вихревым конфигурациям приведем зависимости количества панкейков в слое от номера слоя (отсчитывается со стороны, наиболее удаленной от магнита; рис. 6). Данные зависимости для неоднородного поля оказываются растущими, в то время как для однородного поля число панкейков в слое не зависит от его номера.
Видно, что в неоднородном поле вихревые нити проникают в образец неравномерно, с образованием большого количества неполных вихревых нитей в верхней части образца. При этом в бездефектном образце вихри в однородном поле заполняют сразу весь образец. Неравномерное заполнение образца вихрями приводит к зависимости намагниченности от толщины образца. Для характеристики заполнения сверхпроводника вихрями введем отношение намагниченности всего образца к намагниченности насыщения. Намагниченность каждого слоя рассчитывается как $-4 \pi M=n(i) \Phi_{0}-H(i), n(i)-$ концентрация панкейков в слое $i, \Phi_{0}-$ квант магнитного потока. Намагниченностью насыщения $M_{s}$ называ- 


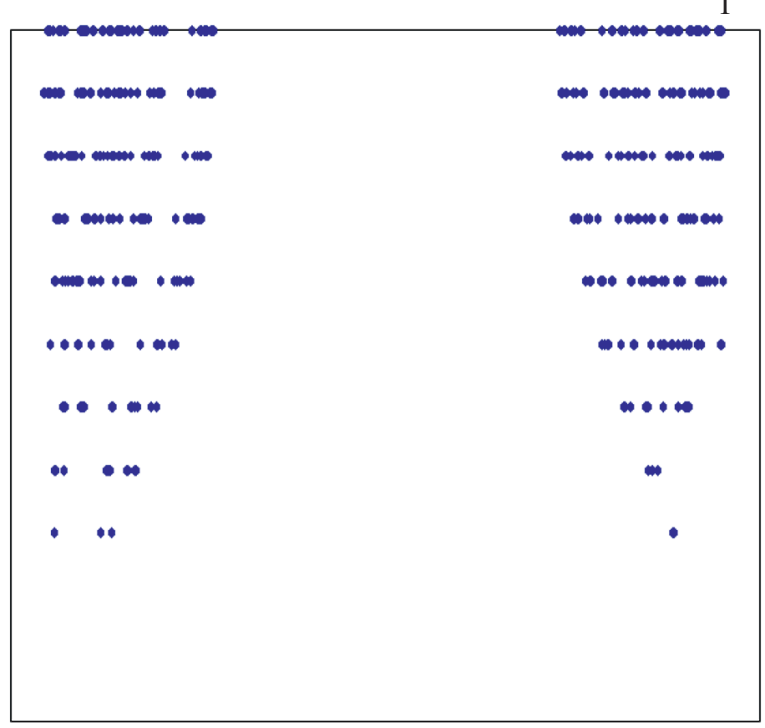

3

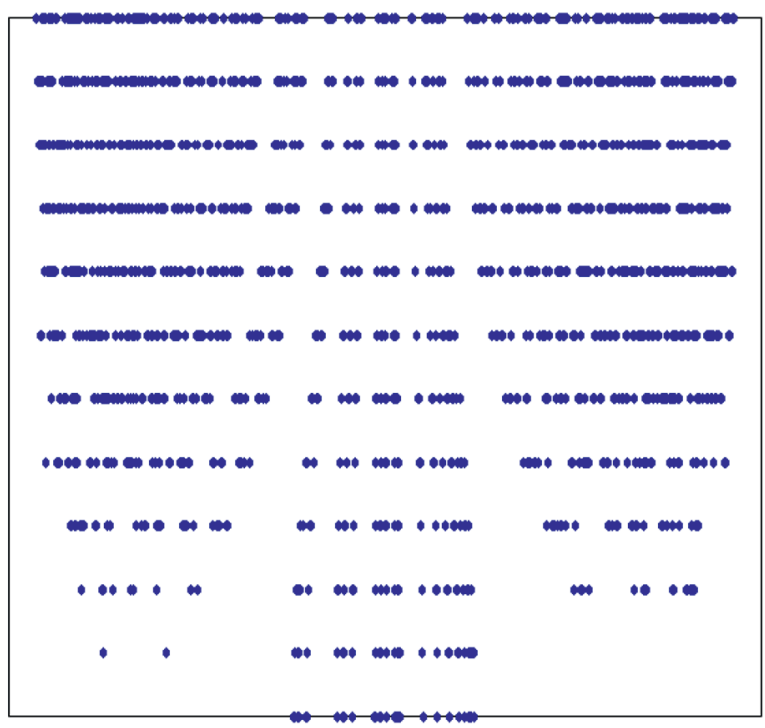

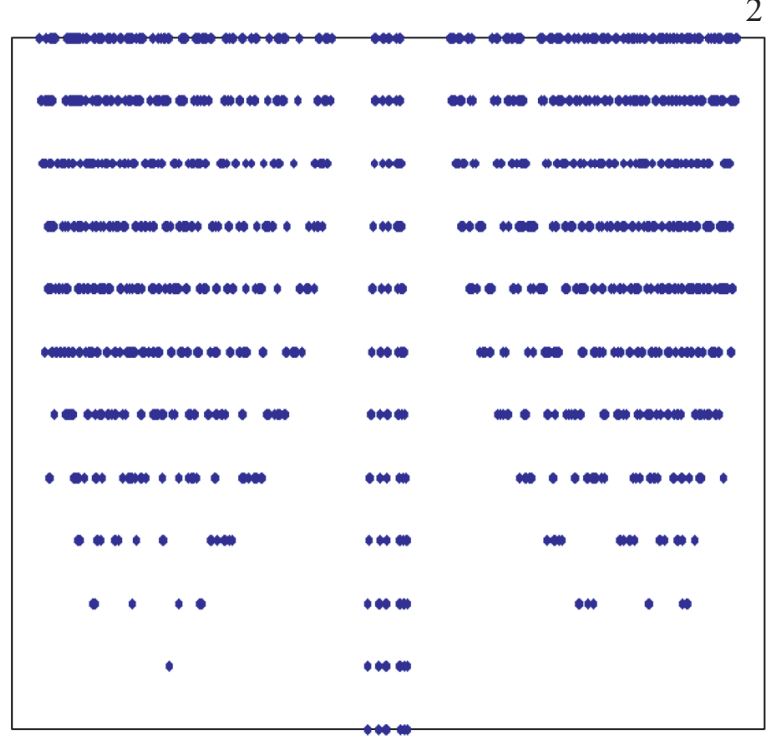

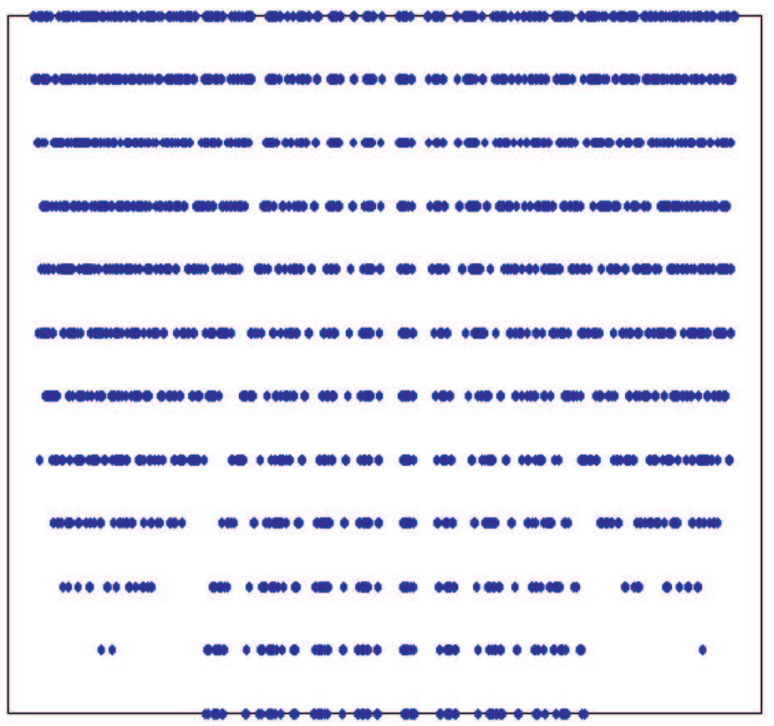

Рис. 6. Проникновение магнитного потока в чистый образец (неоднородное поле). Показаны конфигурации вихрей, возникающие при перемагничивании. 1) $-H=300 \mathrm{Gs}, 2)-H=355 \mathrm{Gs}, 3)-H=370 \mathrm{Gs}, 4)-H=400 \mathrm{Gs}$.

ется намагниченность в ситуации, когда сверхпроводник полностью заполнен вихрями (равновесная конфигурация) и энергия системы соответствует минимуму при данном внешнем поле. Если концентрация панкейков меньше равновесной, то $M / M_{s}>1$. Заметим, что данное отношение приближается к 1 только в сильном, однородном поле. Таким образом, можно сказать, что неоднородное поле приводит к эффективному пиннингу вихревых нитей на границе сверхпроводника. На рис. 8 показаны зависимости $M / M_{s}$ от количества сверхпроводящих слоев в образце. В бездефектном случае при высоких полях $(H=H(1)=800 \mathrm{Gs})$ намагниченность как в однородном, так и в неоднородном поле сразу выходит на насыщение, соответствующая зависимость намагниченности от числа слоев практически горизон- тальна. При низких полях ( $H=450 \mathrm{Gs})$ как в дефектном, так и в бездефектном образце (в дефектном также и при высоких полях) намагниченность быстрее выходит на насыщение в однородном поле (из-за формирования неполных вихревых нитей в неоднородном поле). При числе слоев $N_{\mathrm{L}} \sim 3$ намагниченности образца в однородном и неоднородном поле в пределах погрешности расчета совпадают, что позволяет оценить предельную толщину $(\sim 1 \mu \mathrm{m})$, при которой образец ведет себя в неоднородном поле как тонкая пленка.

Проникновение в образец неоднородного магнитного поля происходит через формирование неполных вихревых нитей и начинается с верхней части образца. В бездефектном образце вихри заполняют образец полностью уже при поле $H>400 \mathrm{Gs}$. 

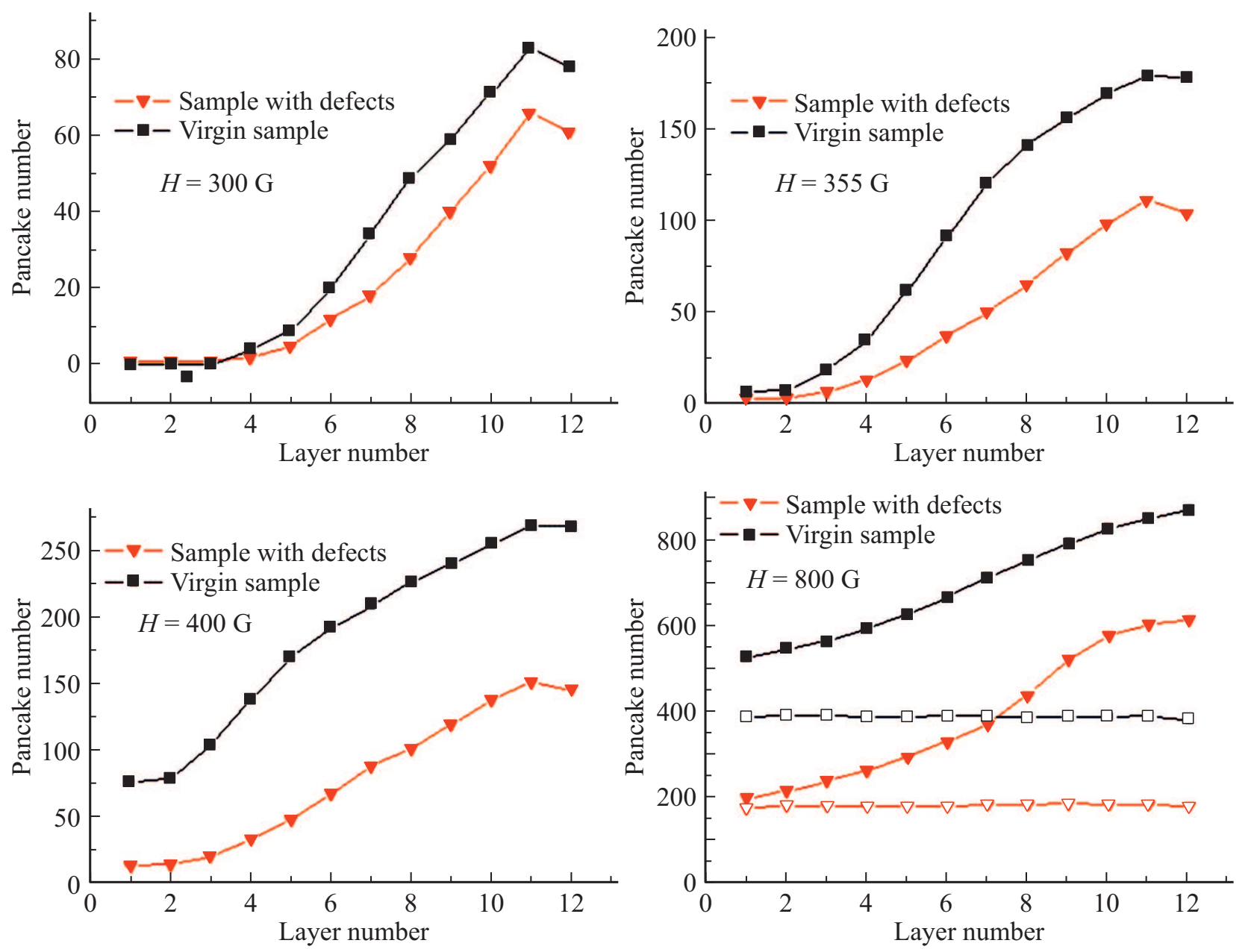

Рис. 7. Зависимости числа пэнкейков в слое от номера слоя. Сплошные символы относятся к неоднородному полю, пустые - к однородному. $H$ - поле, соответствующее слою, наиболее удаленному от магнита.
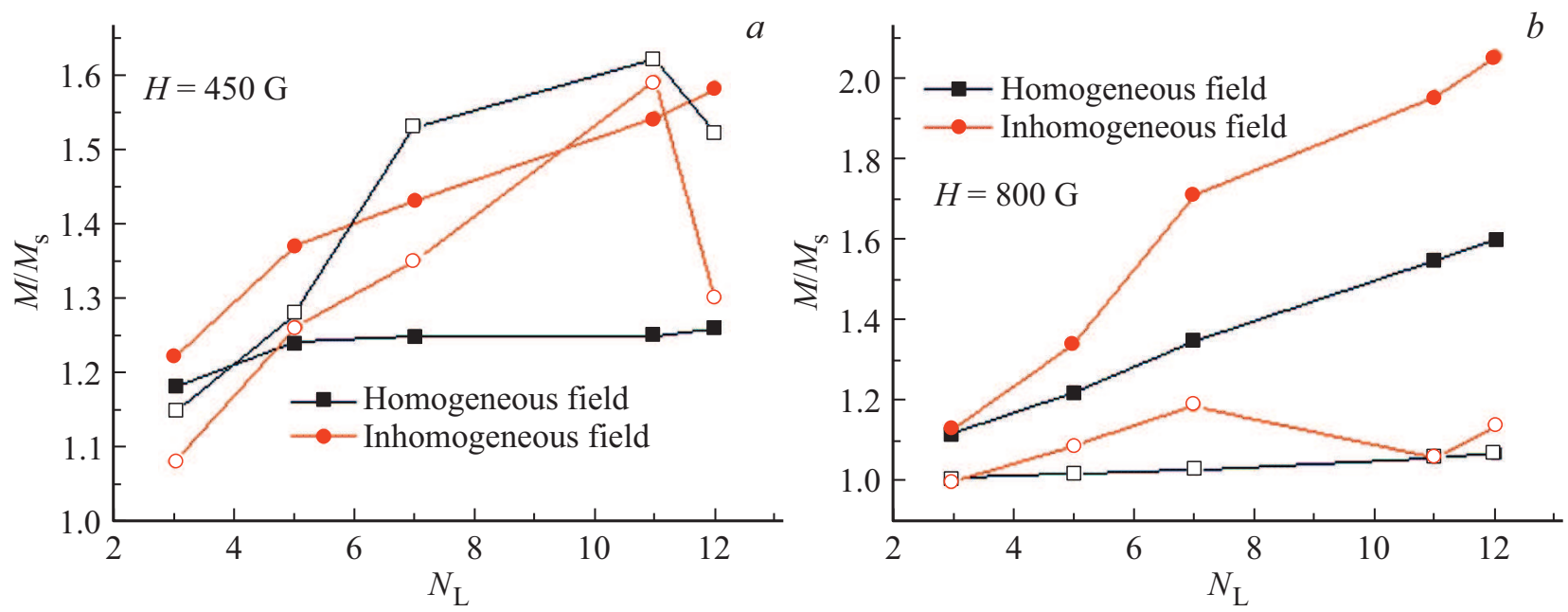

Рис. 8. Отношение намагниченности образца к намагниченности насыщения $M_{s}$ при данном внешнем магнитном поле $(H-$ поле, в котором находится нижний слой) в зависимости от числа слоев $\left.\left.N_{\mathrm{L}} . a\right) H=450 \mathrm{Gs}, b\right) H=800 \mathrm{Gs}$. Заполненные кружки относятся к дефектному образцу, пустые - к бездефектному. В низких полях даже в бездефектном случае вихри не полностью заполняют сверхпроводник, что означает эффективный пиннинг вихревых нитей на границе. 

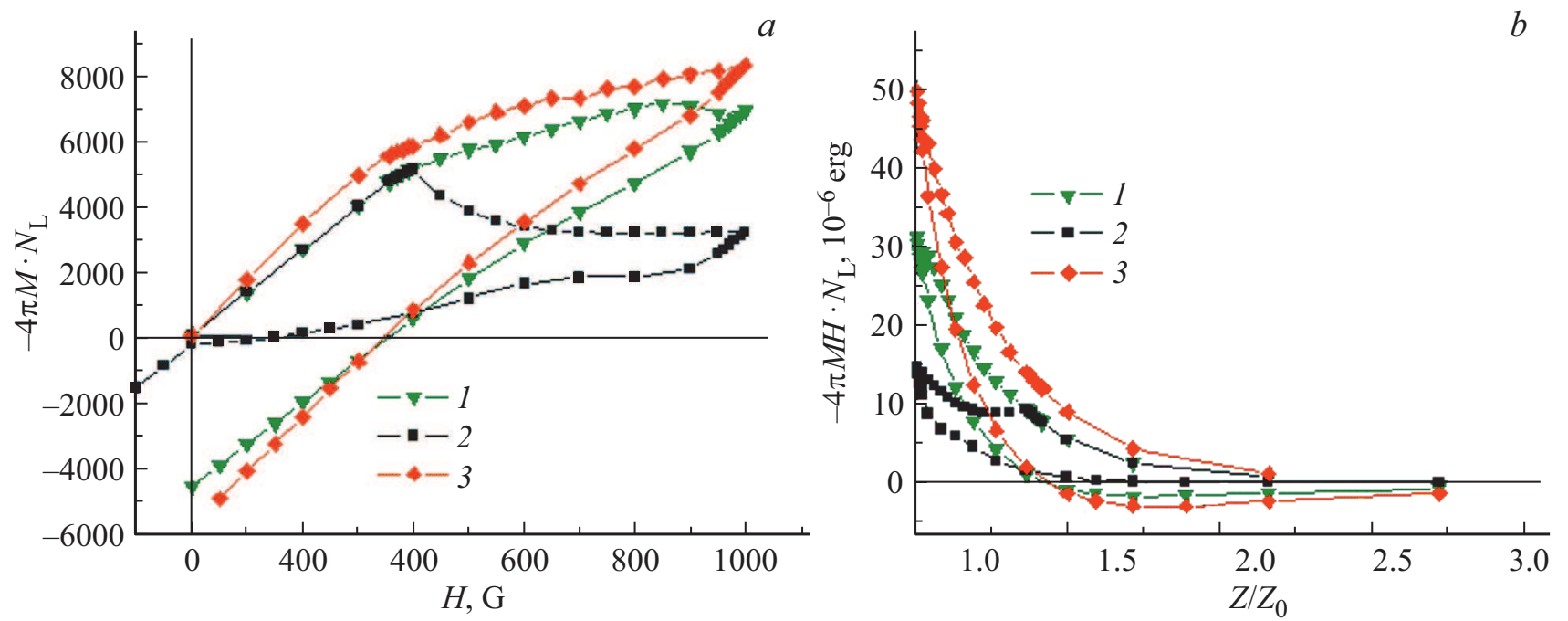

Рис. 9. а) Кривые намагниченности, $1-N_{\mathrm{d}}=300$, inh $=0.02,2-N_{\mathrm{d}}=10$, inh $=0.02,3-N_{\mathrm{d}}=300$, inh $\left.=0.08, b\right)$ энергия в магнитном поле в зависимости от расстояния до магнита. Зависимости показаны при нескольких значениях неоднородности поля. Кривая 1 по параметрам дефектов соответствует кривой 2 на рис. $2, a$. Видно, что, в отличие от кривой 2 , данная кривая идет вверх вследствие пиининга неполных вихревых нитей на границе.

Полученные распределения захваченного магнитного потока в бездефектном образце качественно совпадают с измеренными в работе [32]. Распределения захваченного потока в дефектном образце близки к экспериментальным [32] только в приграничных областях, т.к. при значениях магнитного поля, использованных в расчете, вихри в процессе перемагничивания еще не доходят до центра образца, где таким образом присутствует область, свободная от вихрей. Для более детального сравнения с экспериментом следует сделать расчет при более высоких магнитный полях, для образцов большего размера и при более высокой температуре (в настоящей работе для исключения эффектов, связанных с тепловыми флуктуациями, расчет сделан при температуре жидкого гелия). Отметим, что формирование неполных вихревых нитей означает появление параллельной слоям компоненты магнитного поля, связанной с формированием джозефсоновской струны. Эффекты, обусловленные рождением джозефсоновских вихрей в слоистом сверхпроводнике, исследованы в работах $[12,13,33]$.

Предположим, что указанной зависимостью $H(i)$ может быть описано поле постоянного магнита. Проанализируем намагниченность стопки сверхпроводящих плоскостей в неоднородном поле, создаваемом магнитом. На рис. 9 показаны кривые намагниченности и зависимости энергии в магнитном поле от расстояния до магнита для чистого и дефектного сверхпроводника. Число дефектов в дефектном образце $N_{\mathrm{d}}=300$, что соответствует концентрации дефектов $c=2 \cdot 10^{9} \mathrm{~cm}^{-2}$.

Зависимость энергии для трехмерного дефектного образца имеет один минимум, возникающий при движении образца от магнита, для чистого образца минимум реализуется при движении сверхпроводника к магниту.

\section{4. Заключение}

Методом Монте-Карло выполнено моделирование вихревой решетки в слоистом ВТСП, помещенном в неоднородное магнитное поле. Получены вихревые конфигурации, возникающие в стопке сверхпроводящих плоскостей, проанализирован случай чистого и дефектного сверхпроводника. Расчет показывает, что в неоднородном поле наблюдается формирование неполных вихревых нитей на границе, что означает появление параллельной сверхпроводящим слоям компоненты магнитного поля в образце и пиннинг вихревых нитей на границе даже в чистом образце. Наличие пиннинга на границе подтверждается также расчетом кривых намагниченности. С помощью расчетов вихревых конфигураций оценена предельная толщина $(\sim 1 \mu \mathrm{m})$, при которой образец ведет себя в неоднородном поле как тонкая пленка. Рассчитаны зависимости энергии сверхпроводника в магнитном поле в зависимости от расстояния до магнита, показано наличие минимумов на данных зависимостях, соответствующих устойчивым положениям образца. Результаты работы могут быть использованы при оценке левитационных свойств реальных образцов с заданным типом дефектов.

\section{Финансирование работы}

Исследование выполнено за счет гранта Российского научного фонда (проект № 17-19-01527).

\section{Конфликт интересов}

Авторы заявляют, что у них нет конфликта интересов. 


\section{Список литературы}

[1] Mirela Dragomir, Qianli Ma, J. Patrick Clancy, Amirreza Ataei, Paul A. Dube, Sudarshan Sharma, Ashfia Huq, Hanna A. Dabkowska, Louis Taillefer, Bruce D. Gaulin. Phys. Rev. Mater. 4, 114801 (2020).

[2] Derrick Van Gennep, Abdelwahab Hassan, Huiqian Luo, Mahmoud Abdel-Hafiez. Phys. Rev. B 101, 235163 (2020).

[3] Z. Deng, M. Miki, K. Tsuzuki, B. Felder, R. Taguchi, N. Shinohara, M. Izumi. IEEE Trans. Appl. Supercond. 21, 3, 1180 (2011).

[4] Yujun Dong, Jiahui Zhu, Defu Wei, Wei Chen, Qingqing Du, Ke Zhang, Panpan Chen, Hao Jiang, Shuai Wang, Tie Guo, Kaizhong Ding. Cryogenics 112, 103195 (2020).

[5] M. Badakhshan, S.M. Mousavi. Physica C 547, 51 (2018).

[6] Sait Barüs. Güner, Murat Abdioglu, Kemal Oztürk, Sükrü Çelik. J. Alloys Comp. 822, 153637 (2020).

[7] Jing Jiang, Yuhang Li, Ju wang, Lifeng Zhao, Yong Zhang, Yong Zhao. doi: https://doi.org/10.1016/j.physc.2019.1353582

[8] Z.M. Zhao, J.M. Xu, X.Y. Yuan, C.P. Zhang. Physica C 549, 154 (2018).

[9] J.R. Clem. Phys. Rev. B 43, 7837 (1991).

[10] J.R. Clem, M.W. Coffey. Phys. Rev. B 42, 10, 6209 (1990).

[11] J.R. Clem, M.W. Coffey, Zhidong Hao. Phys. Rev. B 44, 6, 2732 (1991).

[12] A.E. Koshelev. Phys. Rev. B 71, 174507 (2005).

[13] A.E. Koshelev. Phys. Rev. B 48, 2, 1180 (1993).

[14] A. Buzdin, I. Baladié. Phys. Rev. Lett. 88, 14, 147002 (2002).

[15] S.J. Bending, Matthew J.W. Dodgson. J. Phys.: Condens. Matter 17, R955 (2005).

[16] A.V. Samokhvalov, A.S. Mel'nikov, A.I. Buzdin. Phys. Rev. B 85, 184509 (2012).

[17] I. Rudnev, D. Abin, M. Osipov, S. Pokrovskiy, Y. Ermolaev, N. Mineev. Phys. Proc. 65, 141 (2015).

[18] M. Osipov, A. Starikovskii, D. Abin, I. Rudnev. Supercond. Sci. Technol. 32, 5, 054003 (2019).

[19] S.V. Pokrovskii, O.B. Mavritskii, A.N. Egorov, N.A. Mineev, A.A. Timofeev, I.A. Rudnev. Supercond. Sci. Technol., 32, 7, 075008, (2019).

[20] I. Anischenko, S. Pokrovskii, I. Rudnev, M. Osipov. Supercond. Sci. Technol. 32, 105001 (2019).

[21] I. Rudnev, M. Osipov, S. Pokrovskii, A. Podlivaev. Mater. Res. Exp. 6, 3, 036001 (2019).

[22] A. Sanchez, C. Navau. Phys. Rev. B 64, 214506 (2001).

[23] C. Navau, A. Sanchez. Phys. Rev. B 64, 214507 (2001).

[24] A.B. Riise, T.H. Johansen, H. Bratsberg, M.R. Koblischka, Y.Q. Shen. Phys. Rev. B 60, 13, 9855 (1999).

[25] M.J. Qin, G. Li, H.K. Liu, S.X. Dou, E.H. Brandt. Phys. Rev. B 66, 024516 (2002).

[26] V.A. Kashurnikov, A.N. Maksimova, I.A. Rudnev. Phys. Solid State 56, 5, 894 (2014).

[27] I.A. Rudnev, D.S. Odintsov, V.A. Kashurnikov. Phys. Lett. A 372, 3934 (2008).

[28] W.E. Lawrence, S. Doniach. In: Proceedings LT 12. Kyoto (1970) / Ed. E. Kanda. Keigaku, Tokyo (1971). 361 p.

[29] S. Tyagi,Y.Y. Goldschmidt. Phys. Rev. B 70, 024501 (2004).
[30] Y.Y. Goldschmidt, S. Tyagi. Phys. Rev. B 71, 014503 (2005).

[31] G. Blatter, M.V. Feigel'man, V.B. Geshkenbein, A.I. Larkin, V.M. Vinokur. Rev. Mod. Phys. 66, 4, 1125 (1994).

[32] Y.-N. Wang, W.-M. Yang, P.-T. Yang, C.-Y. Zhang, J.-L. Chen, Li-J. Zhang, Li Chen. Physica C 542, 28 (2017).

[33] J.R. Kirtley, V.G. Kogan, J.R. Clem, K.A. Moler. Phys. Rev. B 59, 6, 4343 (1999).

Редактор Т.Н. Василевская 